\title{
Recent Update of Korea Acute Myocardial Infarction Registry (KAMIR)
}

\author{
Doo Sun Sim and Myung Ho Jeong
}

\begin{abstract}
Korea Acute Myocardial Infarction Registry (KAMIR) is the first nationwide registry and reflects the current therapeutic approaches and management for acute myocardial infarction (AMI) in Korea. The results of KAMIR demonstrated different risk factors and different responses to medical and interventional therapies. The prevalence of hypertension, diabetes mellitus and dyslipidemia has increased, but smoking has decreased remarkably. Different pattern of dyslipidemia was demonstrated. The KAMIR score was better than TIMI and GRACE scores in the prediction of long-term mortality in AMI patients. Triple antiplatelet therapy, consisting of aspirin, clopidogrel and cilostazol, was effective in preventing adverse clinical outcomes after percutaneous coronary intervention (PCl). Prasugrel and ticagrelor could not reduce ischemic events, but increased bleeding risk in Korean patients. Therefore, we may recommend low dose prasugrel and ticagrelor compared with Western patients. Statin therapy was effective in Korean AMI patients, including those with very low levels of low density cholesterol and spasm-induced AMI. Statin and ezetimibe was effective in high-risk AMI patients, such as diabetes, old age and systolic heart failure. PCI and its success rates of ST-elevation myocardial infarction (STEMI) are $96.7 \%$ and $99.4 \%$, and those of non-ST-elevation myocardial infarction (NSTEMI) $82.7 \%$ and $99.5 \%$, respectively. Drug-eluting stents were effective and safe in AMI patients with no increased risk of stent thrombosis in Korean patients. KAMIR will provide new therapeutic strategies and effective methods for secondary prevention of $\mathrm{AMI}$ and new guidelines for Asian patients.
\end{abstract}

KEY WORDS: myocardial infarction, percutaneous coronary intervention, risk factors, statins

\section{Introduction}

Acute myocardial infarction (AMI) is a leading cause of mortality and one of the major challenges facing health care systems in the world. The Korea Acute Myocardial Infarction Registry (KAMIR) is the first nationwide registry designed to track clinical outcomes of patients with AMI. KAMIR has enabled us to prospectively follow the incidence and characteristics of AMI and to perform comprehensive analyses of AMI in the Korean population. More than 61,000 patients have been registered in KAMIR since 2005 and a total of 178 papers, including 166 papers have been published in Science Citation Index journals so far.

KAMIR study demonstrated different etiologies and risk factors, different responses to anti-platelet therapies, renin-angiotensin system inhibitors and statin, different methods of interventional treatments from Western patients ${ }^{1-6)}$. KAMIR study reflects the current therapeutic approaches and management for AMI in Korea. The results of KAMIR could help clinicians to

Dr. Myung Ho Jeong is a principal investigator of Korea Acute Myocardial Infarction Registry, The Heart Research Center of Chonnam National University Hospital, 671 Jaebong-ro, Dong-gu, Gwangju, 501-757 Korea J-STAGE Advance Published Date: February 10, 2017

doi: 10.7793/jcoron.23.001 predict the prognosis of their patients and identify better diagnostic and treatment tools to improve the quality of care.

In this review article, we describe different aspects of risk factors, risk score systems, medical and interventional managements of Korean AMI patients compared with Western patients.

\section{General Trends of KAMIR}

ST-elevation myocardial infarction (STEMI) has been gradually decreased and non-ST-elevation myocardial infarction (NSTEMI) increased in Korea. The ratio of STEMI/NSTEMI in Korea was reversed in 2012 with higher incidence of NSTEMI than STEMI ${ }^{1)}$. Intensive medical treatment including high-intensity statin and new anti-platelet therapy, and percutaneous coronary intervention (PCI) using second generation drug-eluting stents (DES) are commonly used in the treatment of Korean AMI patients ${ }^{2)}$.

\section{Risk Factors}

Risk factors of Korean AMI patients are different from those of Western patients. Risk factors of hypertension, diabetes mellitus and dyslipidemia have been increased, but smoking has been decreased remarkably in Korea. Previously smoking was the most important risk factor in Korean AMI patients. The history of smoking has been decreased from $62.0 \%$ in 2006 to $44.6 \%$ in 
2013 in STEMI patients and from $51.1 \%$ in 2006 to $34.5 \%$ in NSTEMI patients ${ }^{1)}$. Smoking in female patients is one of the predictive factors of major adverse cardiac events (MACE) according to multivariate logistic regression analysis ${ }^{7}$. The pattern of dyslipidemia in Korean patients is different from that of Western patients. In Korean patients, the mean value of low density lipoprotein (LDL)-cholesterol is $113.8 \pm 38.7 \mathrm{mg} / \mathrm{dl}$ in STEMI, $109.6 \pm 39.6 \mathrm{mg} / \mathrm{dl}$ in NSTEMI, which is not so high compared with Western patients. The mean level of triglycerides (TG) was $139.8 \pm 124.5 \mathrm{mg} / \mathrm{dl}$ in STEMI and $128.9 \pm 114.9 \mathrm{mg} / \mathrm{dl}$ in NSTEMI, and that of high density lipoprotein (HDL)-cholesterol was $42.6 \pm 12.3 \mathrm{mg} / \mathrm{dl}$ in STEMI and $43.1 \pm 12.7 \mathrm{mg} / \mathrm{dl}$ in NSTEMI in Korean patients ${ }^{2)}$. About half of the AMI patients have low HDL-C and one quarter of AMI patients have high TG in Korea. In these patients, statin therapy was not effective in the secondary prevention of AMI during one-year clinical follow-up. Therefore, differential benefit of statin was demonstrated in Korean patients ${ }^{5}$. TG/HDL-C ratio higher than 3.35 was associated with development of overall $\mathrm{MACE}^{8}$. Obesity has been decreased from $41.2 \%$ to $35.0 \%$ in STEMI and from $41.1 \%$ to $35.1 \%$ in NSTEMI between 2006 and 2013 in Korea ${ }^{1)}$. Low level of HDL-C in overnight fasting status is associated with high in-hospital mortality in STEMI patients, not in NSTEMI patients $^{9)}$. Two target goals of LDL-C were compared in Korean AMI patients: $50 \%$ reduction from baseline vs. $<70 \mathrm{mg} / \mathrm{dl}$. More than $50 \%$ reduction of LDL-C from baseline was associated with reduction of MACE during 2-year clinical follow-up, but not in target LDL-C goal $<70 \mathrm{mg} / \mathrm{dl}^{10)}$.

AMI patients with low $(<100 / 60 \mathrm{mmHg})$ and high $(>170 / 100$ $\mathrm{mmHg}$ ) blood pressures in KAMIR are associated with MACE. Therefore J-curve phenomenon was observed in the blood pressure of Korea patients ${ }^{11)}$. Hypoglycemia ( $\left.<70 \mathrm{mg} / \mathrm{dl}\right)$ and hyperglycemia $(\geq 260 \mathrm{mg} / \mathrm{dl})$ are associated with higher one-month mortality in diabetic and non-diabetic patients. Especially, hypoglycemia in poorly controlled type 2 diabetic patients predicts one-month mortality in Korean AMI patients ${ }^{12)}$. Under-nutrition at admission influences on clinical outcome in AMI and is associated with high post-AMI complications and high in-hospital mortality. According to Geriatric Nutritional Risk Index, under-nutrition was present in $18 \%$ of AMI patients ${ }^{13}$.

\section{Risk Scores [KAMIR Scores]}

The KAMIR score was proposed to be a predictor of the prognosis of AMI patients, and KAMIR score was compared with Western score systems, such as Thrombolysis In Myocardial Infarction (TIMI) and Global Registry of Acute Coronary Events (GRACE) scores in the prediction of long-term mortality. New score system for NSTEMI include TIMI risk index, Killip class and serum creatinine ${ }^{14)}$. This score systems predict more precise- ly than Western scores. Hospital discharge score system for all AMI patients was composed of age, Killip class, PCI, serum creatinine, left ventricular ejection fraction $<40 \%$ and admission hyperglycemia $>180 \mathrm{mg} / \mathrm{dl}$. KAMIR score was better than GRACE score in the prediction of one-year mortality after discharge ${ }^{15)}$. A new score system including biomarkers (high-sensitivity-C-reactive protein [hs-CRP], glomerular filtration rate, $\mathrm{N}$-terminal pro-brain natriuretic peptide) predicts all cause-mortality in NSTEMI patients in Korean patients. CHA2DS2-VASc scoring system can be used as a screening method for high-risk AMI patients and high score predicts in-hospital mortality and complications ${ }^{16)}$. More than $80 \%$ reduction of hs-CRP is related to secondary prevention in one-year survivors after AMI in Ko$\mathrm{rea}^{17)}$. Simple ACEF (age, creatinine, ejection fraction) score can predict one-year mortality in 30-day survivors who underwent PCI after $\mathrm{AMI}^{18)}$

\section{Medical Treatment}

Triple anti-platelet therapy including aspirin, clopidogrel and cilostazol, is associated with lower development of MACE compared with dual anti-platelet therapy in AMI patients who underwent primary PCI, especially in diabetic patients ${ }^{19)}$. Standard or low dose maintenance dosage of prasugrel $(10 \mathrm{mg}$ and $5 \mathrm{mg}$ ) is associated with lower level of platelet reactivity unit compared with triple anti-platelet therapy in STEMI patients ${ }^{20}$. Thus, low dose $5 \mathrm{mg}$ prasugrel can be used in Korean patients with STEMI. In Western countries, ticagrelor reduces ischemic events without increasing risk of bleeding complications. However, ticagrelor does not reduce MACE but increases TIMI major and minor bleeding risks in Korean patients ${ }^{3)}$. Therefore, reduced dosage of ticagrelor may be beneficial in Korean AMI patients.

Early statin therapy in AMI patients who have extremely low LDL-C ( $<70 \mathrm{mg} / \mathrm{dl})$ was effective in the reduction of cumulative incidence of $\mathrm{MACE}^{21)}$. Early statin therapy in statin-naïve patients with cardiogenic shock from the KAMIR who underwent revascularization was associated with lower in-hospital mortality and MACE at 12 months $^{22)}$. Statin therapy was effective in spasm induced AMI patients in terms of MACE-free survival ${ }^{23)}$. Statin plus ezetimibe could not reduce MACE compared with high-intensive statin in Korea AMI patients. In subgroup analysis, statin plus ezetimibe was effective in high risk patients, such as diabetes mellitus, old age and low left ventricular ejection fraction $^{24)}$.

In Korean AMI patients, angiotensin receptor blocker (ARB) is better than angiotensin converting enzyme inhibitor (ACEI) in STEMI patients, including subgroups of old age, female, diabetes, high Killip class, multi-vessel disease and the left anterior descending artery as the infarct-related artery ${ }^{4)}$. Surmountable $\mathrm{ARB}$, such as valsartan, candesartan, irbesartan, telmisartan and 
olmesartan, is better than surmountable ARB (losartan, eprosar$\tan )$ in the development of MACE in Korean patients ${ }^{25)}$. Renin-angiotensin system inhibitor was effective in STEMI patients with normal and abnormal left ventricular function who underwent successful late $\mathrm{PCI}^{26)}$.

\section{Interventional Treatment}

Door-to-balloon time in STEMI patients has been reduced to less than 60 minutes in major primary PCI centers participated in KAMIR, but one-month mortality has not been decreased. Total ischemic time less than 180 minutes is associated with onemonth mortality ${ }^{27)}$. Thus, shortening of symptom to door time may be more important than shortening of door to balloon time in Korea. Pharmacoinvasive strategy in STEMI patients who underwent primary PCI has comparable clinical outcomes to patients who underwent primary PCI. If PCI-related delay is longer than $\approx 100$ minutes, pharmacoinvasive treatment can be recommended in STEMI patients ${ }^{28)}$. Early elective PCI within 48 hours after successful thrombolytic therapy for acute STEMI was associated with a more favorable clinical outcome than patients who underwent PCI later ${ }^{29)}$. PCI in stable, early latecomers with STEMI presenting 12 to 72 hours after symptom onset reduced mortality and the incidence of death/myocardial infarction (MI) at 12 months $^{30)}$. Multi-vessel PCI in multi-vessel coronary artery disease is associated with higher MACE-free survival and death/ MI-free survival compared with culprit-only PCI in NSTEMI patients $^{31)}$. About $52 \%$ of STEMI patients have non-infarct related coronary artery disease and one-month mortality is higher in STEMI patients with multi-vessel lesions in Korea ${ }^{32}$. Three-year clinical outcomes of staged PCI are better than ad hoc or culprit-only PCI in STEMI patients with multi-vessel diseases ${ }^{33)}$.

Rates of PCI were $96.7 \%$ in STEMI and $82.7 \%$ in NSTEMI, and the success rates were $99.4 \%$ and $99.5 \%$, respectively, which were relatively higher in Korea than in Western countries ${ }^{2)}$. Patients with culprit left main coronary artery stenosis had higher in-hospital mortality than patients with non-culprit left main coronary artery, but had similar clinical outcome during a 12-month follow-up ${ }^{34)}$. Penetration rate of DES is higher than 90\% (97\% currently) in Korea. The event rates are lower in DES implanted group compared with bare metal stent (BMS) group by reduction of repeat intervention, not increasing mortality, MI and stent thrombosis ${ }^{6}$. DES in large vessels in AMI was associated with lower need for repeat revascularization compared to BMS without compromising the overall safety over the course of one-year follow-up ${ }^{35)}$. Zotarolimus-and everoliumus-eluting stents have comparable clinical outcomes in STEMI patients with chronic kidney disease (CKD) undergoing primary $\mathrm{PCI}^{36}$. In the management of NSTEMI with CKD, deferred invasive group is associated with higher death/MI-free survival rate than early invasive group $^{37)}$. In octogenarian patients with NSTEMI, there was no difference in MACE-free survival between early and delayed intervention group ${ }^{38)}$. In NSTEMI patients, immediate PCI less than 4 hours was associated with lower major bleeding and shorter hospital stay compared with non-immediate PCI group in Korea. However, MACE were not different between immediate and non-immediate $\mathrm{PCI}^{39)}$. These results were different from Western countries.

In octogenarian AMI patients, DES is associated with lower stent thrombosis rate compared with BMS and all major cardiac events are not different between DES and BMS groups ${ }^{40)}$. In patients with metabolic syndrome and AMI, patients-oriented composite events (POCE) were not different between Resolute zotarolimus-eluting stents versus everolimus-eluting stents during after propensity score-matched analysis ${ }^{41)}$.

Use of the intra-aortic balloon pump for resuscitated patients with AMI complicated by cardiac arrest could not reduce cumulative incidence of mortality compared with control group ${ }^{42)}$. Intravascular ultrasound (IVUS) guided PCI is performed in about $20 \%$ of AMI patients, but cumulative survival is not different from non-IVUS group ${ }^{43)}$. Thrombus aspiration during primary PCI could not reduce MACE in STEMI patients, but thrombus aspiration was associated with better clinical outcome in subgroups, such as use of platelet glycoprotein IIb/IIIa receptor blocker and lesion location in left anterior descending artery ${ }^{44)}$. The effect of manual thrombus aspiration on 12-month outcome showed a U-shaped relationship, indicating that the impact of thrombus aspiration may become clinically relevant with longer total ischemic time ${ }^{45}$.

\section{Conclusion}

Risk factors of Korean AMI patients are different from Western countries. Smoking history in Korean patients is relatively higher than Western patients and lipid profiles are different from Western patients. Statin therapy is not effective in AMI patients with high TG and low HDL-C. KAMIR score is better than TIMI or GRACE scores in the prediction of one-year mortality. ARB is better than ACEI in Korea patients; especially insurmountable ARB will be more effective than surmountable ARB. Low dose prasugrel or ticagrelor will be beneficial to prevent bleeding complications. In multi-vessel diseases, staged PCI for STEMI and multi-vessel PCI in NSTEMI can be recommended. DES is safer and more effective than BMS in Korean AMI patients. Octogenarian can be treated by elective PCI using second generation DES. Thrombus aspiration and IVUS-guide PCI can be recommended in selected patients. KAMIR will provide new therapeutic strategies and effective methods for secondary prevention of AMI and ischemic heart failure in Korean patients. KAMIR study will be useful in the establishment of Asian AMI 
guidelines in the future.

All authors declare no conflict of interest.

\section{References}

1) Kook HY, Jeong MH, Oh S, et al; Korea Acute Myocardial Infarction Registry Investigators: Current trend of acute myocardial infarction in Korea (from the Korea Acute Myocardial Infarction Registry from 2006 to 2013) . Am J Cardiol 2014; 114: 1817-1822

2) Kim JH, Chae SC, Oh DJ, et al; Korea Acute Myocardial Infarction-National Institutes of Health Registry Investigators: Multicenter cohort study of acute myocardial infarction in Korea - interim analysis of the Korea Acute Myocardial Infarction Registry-National Institutes of Health registry -. Circ J 2016; 80: 1427-1436

3) Park KH, Jeong MH, Ahn Y, et al; KAMIR-NIH registry investigators: Comparison of short-term clinical outcomes between ticagrelor versus clopidogrel in patients with acute myocardial infarction undergoing successful revascularization; from Korea Acute Myocardial Infarction Registry-National Institute of Health. Int J Cardiol 2016; 215: 193-200

4) Yang JH, Hahn JY, Song YB, et al: Angiotensin receptor blocker in patients with ST segment elevation myocardial infarction with preserved left ventricular systolic function: prospective cohort study. BMJ 2014; 349: g6650

5) Kim KH, Kim CH, Jeong MH, et al; other Korea Acute Myocardial Infarction Registry Investigators: Differential benefit of statin in secondary prevention of acute myocardial infarction according to the level of triglyceride and high density lipoprotein cholesterol. Korean Circ J 2016; 46: 324-334

6) Hong YJ, Jeong MH, Ahn Y, et al: The efficacy and safety of drug-eluting stents in patients with acute myocardial infarction: results from Korea Acute Myocardial Infarction (KAMIR). Int J Cardiol 2013; 163: 1-4

7) Jeong YA, Jeong MH, Jeong HC, et al; Korea Acute Myocardial Infarction Registry (KAMIR) Investigators: Impact of smoking on clinical outcomes in female patients with acute myocardial infarction. Korean Circ J 2015; 45: 22-27

8) Kim JS, Kim W, Woo JS, et al; Korea Acute Myocardial Infarction Registry Investigators: The predictive role of serum triglyceride to high-density lipoprotein cholesterol ratio according to renal function in patients with acute myocardial infarction. PLoS One 2016; 11: e0165484

9) Ji MS, Jeong MH, Ahn YK, et al; Korea Acute Myocardial Infarction Registry Investigators: Impact of low level of high-density lipoprotein-cholesterol sampled in overnight fasting state on the clinical outcomes in patients with acute myocardial infarction (difference between ST-segment and non-ST-segment-elevation myocardial infarction). J Cardiol 2015; 65: 63-70

10) Cho KH, Jeong MH, Park KW, et al; KAMIR (Korea Acute Myocardial Infarction Registry) Investigators: Comparison of the effects of two low-density lipoprotein cholesterol goals for secondary prevention after acute myocardial infarction in real-world practice: $\geq 50 \%$ reduction from baseline versus $<70 \mathrm{mg} / \mathrm{dL}$. Int J Cardiol 2015; 187: $478-485$
11) Cho JY, Jeong MH, Ahn Y, et al; Korea Acute Myocardial Infarction Registry Investigators: Impact of high admission blood pressure without history of hypertension on clinical outcomes of patients with acute myocardial infarction: from Korea Acute Myocardial Infarction Registry. Int J Cardiol 2014; 172: e54-58

12) Lee SA, Cho SJ, Jeong MH, et al; KAMIR/KorMI Registry: Hypoglycemia at admission in patients with acute myocardial infarction predicts a higher 30-day mortality in patients with poorly controlled type 2 diabetes than in well-controlled patients. Diabetes Care 2014; 37: 2366-2373

13) Yoo SH, Kook HY, Hong YJ, et al: Influence of undernutrition at admission on clinical outcomes in patients with acute myocardial infarction. J Cardiol 2016 doi: 10.1016/j.jjcc.2016.05.009 [Epub ahead of print]

14) Kim HK, Jeong MH, Ahn Y, et al; other Korea Acute Myocardial Infarction Registry Investigators; Korea Acute Myocardial infarction Registry (KAMIR) Study Group of Korean Circulation Society: A new risk score system for the assessment of clinical outcomes in patients with non-ST-segment elevation myocardial infarction. Int J Cardiol 2010; 145: 450-454

15) Kim HK, Jeong MH, Ahn Y, et al; other Korea Acute Myocardial Infarction Registry Investigators: Hospital discharge risk score system for the assessment of clinical outcomes in patients with acute myocardial infarction (Korea Acute Myocardial Infarction Registry [KAMIR] score). Am J Cardiol 2011; 107: 965-971. e1

16) Jeong YW, Jeong MH, Kim SS, et al: CHA2DS2-VASc scoring system as an initial method for screening high-risk patients in acute myocardial infarction. Int J Cardiol 2014; 174: 777-780

17) Kim HK, Jeong MH, Seo HW, et al; Korea Acute Myocardial Infarction Registry Investigators: Clinical impacts of high-sensitivity C-reactive protein reduction for secondary prevention in Asian patients with one-year survivor after acute myocardial infarction. Int J Cardiol 2015; 193: 20-22

18) Lee JH, Bae MH, Yang DH, et al; Korea Acute Myocardial Infarction Registry Investigators: Prognostic value of the age, creatinine, and ejection fraction score for 1-year mortality in 30-day survivors who underwent percutaneous coronary intervention after acute myocardial infarction. Am J Cardiol 2015; 115: 1167-1173

19) Chen KY, Rha SW, Li YJ, et al; Korea Acute Myocardial Infarction Registry Investigators: Triple versus dual antiplatelet therapy in patients with acute ST-segment elevation myocardial infarction undergoing primary percutaneous coronary intervention. Circulation 2009; 119: $3207-3214$

20) Park KH, Jeong MH, Lee KH, et al: Comparison of peri-procedural platelet inhibition with prasugrel versus adjunctive cilostazol to dual anti-platelet therapy in patients with ST segment elevation myocardial infarction. J Cardiol 2014; 63: 99-105

21) Lee KH, Jeong MH, Kim HM, et al; KAMIR (Korea Acute Myocardial Infarction Registry) Investigators: Benefit of early statin therapy in patients with acute myocardial infarction who have extremely low low-density lipoprotein cholesterol. J Am Coll Cardiol 2011; 58: 1664-1671

22) Sim DS, Jeong MH, Cho KH, et al; other Korea Acute Myocardial Infarction Registry (KAMIR) Investigators: Effect of early statin treatment in patients with cardiogenic shock complicating acute myo- 
cardial infarction. Korean Circ J 2013; 43: 100-109

23) Piao ZH, Jeong MH, Li Y, et al; other Korea Acute Myocardial Infarction Registry (KAMIR) Investigators: Benefit of statin therapy in patients with coronary spasm-induced acute myocardial infarction. J Cardiol 2016; 68: 7-12

24) Ji MS, Jeong MH, Ahn YK, et al; other Korea Acute Myocardial Infarction Registry Investigators: Clinical outcome of statin plus ezetimibe versus high-intensity statin therapy in patients with acute myocardial infarction propensity-score matching analysis. Int $\mathrm{J}$ Cardiol 2016; 225: 50-59

25) Jeong HC, Jeong MH, Ahn Y, et al; Korea Acute Myocardial Infarction Registry Investigators: Comparative assessment of angiotensin II type 1 receptor blockers in the treatment of acute myocardial infarction: surmountable vs. insurmountable antagonist. Int J Cardiol 2014; 170: 291-297

26) Park H, Kim HK, Jeong MH, et al: Clinical impacts of inhibition of renin-angiotensin system in patients with acute ST-segment elevation myocardial infarction who underwent successful late percutaneous coronary intervention. J Cardiol 2017; 69: 216-221

27) Kim HK, Jeong MH, Ahn Y, et al; other Korea Acute Myocardial Infarction Registry Investigators: Relationship between time to treatment and mortality among patients undergoing primary percutaneous coronary intervention according to Korea Acute Myocardial Infarction Registry. J Cardiol 2017; 69: 377-382

28) Sim DS, Jeong MH, Ahn Y, et al; Korea Acute Myocardial Infarction Registry (KAMIR) Investigators: Pharmacoinvasive strategy versus primary percutaneous coronary intervention in patients with ST-segment-elevation myocardial infarction: a propensity score-matched analysis. Circ Cardiovasc Interv 2016; doi: 10.1161/CIRCINTERVENTIONS.115.003508

29) Sim DS, Jeong MH, Ahn Y, et al; Korea Acute Myocardial Infarction Registry (KAMIR) Investigators: Safety and benefit of early elective percutaneous coronary intervention after successful thrombolytic therapy for acute myocardial infarction. Am J Cardiol 2009; 103: $1333-1338$

30) Sim DS, Jeong MH, Ahn Y, et al; Korea Acute Myocardial Infarction Registry (KAMIR) Investigators: Benefit of percutaneous coronary intervention in early latecomers with acute ST-segment elevation myocardial infarction. Am J Cardiol 2012; 110: 1275-1281

31) Kim MC, Jeong MH, Ahn Y, et al; Korea Acute Myocardial Infarction Registry Investigators: What is optimal revascularization strategy in patients with multivessel coronary artery disease in non-ST-elevation myocardial infarction? Multivessel or culprit-only revascularization. Int J Cardiol 2011; 153: 148-153

32) Park DW, Clare RM, Schulte PJ, et al: Extent, location, and clinical significance of non-infarct-related coronary artery disease among patients with ST-elevation myocardial infarction. JAMA 2014; 312: 2019-2027

33) Kim MC, Jeong MH, Park KH, et al: Three-year clinical outcomes of staged, ad hoc and culprit-only percutaneous coronary intervention in patients with ST-segment elevation myocardial infarction and multivessel disease. Int J Cardiol 2014; 176: 505-507

34) Sim DS, Ahn Y, Jeong MH, et al; Korea Acute Myocardial Infarction
Registry (KAMIR) Investigators: Clinical outcome of unprotected left main coronary artery disease in patients with acute myocardial infarction. Int Heart J 2013; 54: 185-191

35) Sim DS, Jeong MH, Ahn Y, et al; Korea Acute Myocardial Infarction Registry (KAMIR) Investigators: Effectiveness of drug-eluting stents versus bare-metal stents in large coronary arteries in patients with acute myocardial infarction. J Korean Med Sci 2011; 26: 521-527

36) Ahmed K, Jeong MH, Chakraborty R, et al; other Korea Acute Myocardial Infarction Registry Investigators: Comparison of zotarolimusand everolimus-eluting stents in patients with ST-elevation myocardial infarction and chronic kidney disease undergoing primary percutaneous coronary intervention. J Cardiol 2014; 64: 273-278

37) Hachinohe D, Jeong MH, Saito S, et al; other Korea Acute Myocardial Infarction Registry Investigators: Management of non-ST-segment elevation acute myocardial infarction in patients with chronic kidney disease (from the Korea Acute Myocardial Infarction Registry). Am J Cardiol 2011; 108: 206-213

38) Piao ZH, Jeong MH, Jin L, et al; other Korea Acute Myocardial Infarction Registry (KAMIR) Investigator: Clinical impact of early intervention in octogenarians with non-ST-elevation myocardial infarction. Int J Cardiol 2014; 172: 462-464

39) Sim DS, Jeong MH, Ahn Y, et al; other Korea Acute Myocardial Infarction Registry (KAMIR) Investigators: Clinical impact of immediate invasive strategy in patients with non-ST-segment elevation myocardial infarction. Int J Cardiol 2016; 221: 937-943

40) Piao ZH, Jeong MH, Li Y, et al; other Korea Acute Myocardial Infarction Registry (KAMIR) Investigators: Comparison of second-generation drug-eluting versus bare-metal stents in octogenarian patients with ST-segment elevation myocardial infarction. Int J Cardiol 2014; 177: 1081-1084

41) Ji MS, Jeong MH, Ahn YK, et al; Korea Acute Myocardial Infarction Registry Investigators: Comparison of Resolute zotarolimus-eluting stents versus everolimus-eluting stents in patients with metabolic syndrome and acute myocardial infarction: propensity score-matched analysis. Int J Cardiol 2015; 199: 53-62

42) Kim HK, Jeong MH, Ahn Y, et al; other Korea Acute Myocardial Infarction Registry Investigators: Clinical outcomes of the intra-aortic balloon pump for resuscitated patients with acute myocardial infarction complicated by cardiac arrest. J Cardiol 2016; 67: 57-63

43) Ahmed K, Jeong MH, Chakraborty R, et al; other Korea Acute Myocardial Infarction Registry Investigators: Role of intravascular ultrasound in patients with acute myocardial infarction undergoing percutaneous coronary intervention. Am J Cardiol 2011; 108: 8-14

44) Hachinohe D, Jeong MH, Saito S, et al; Korea Acute Myocardial Infarction Registry Investigators: Clinical impact of thrombus aspiration during primary percutaneous coronary intervention: results from Korea Acute Myocardial Infarction Registry. J Cardiol 2012; 59: 249-257

45) Sim DS, Jeong MH, Ahn Y, et al; other Korea Acute Myocardial Infarction Registry (KAMIR) Investigators: Manual thrombus aspiration during primary percutaneous coronary intervention: Impact of total ischemic time. J Cardiol 2017; 69: 428-435 